\title{
M. M. Ozek, G. Cinalli, W.J. Maixner (eds) Spina bifida, management and outcome
}

\author{
Springer, Milan Berlin Heidelberg NewYork, 2008, 564 pp
}

\author{
Gianpiero Tamburrini
}

Published online: 11 February 2009

(C) Springer-Verlag 2009

This recently published book on Spina Bifida represents a due update on a disease that has been erroneously considered of relatively low scientific significance in the western world during the last decade. In fact, the common evidence of a decreased birth rate of children with spinal dysraphism in developed countries does not justify a reduction of interest for what still represents a clinical entity with a relevant multidisciplinary impact and burden in the great majority of the world. At first oversight, the reader is impressed by the full-bore dealing of any possible "primary" or "secondary" medical and surgical aspect of the disease and by the even more accurate selection of the contributors based on their subspecialty expertise. Section I, dedicated to general aspects, starts with a very welldocumented historical review of the surgical treatment of spina bifida and a detailed chapter on embryology based on modern research theories, which exemplarily guides through an easier understanding of the subject with a step-by-step collection of pathological specimens, experimental figures and schemes. Pathological clinical and surgical findings are the basis of the classification of spinal dysraphism entities (Chapter 3), Section I ending with an adjourned review of etiological factors and a discussive overview of medical ethics. Section II is dedicated to prenatal diagnosis and prenatal surgical treatment, two fields in which substantial changes have occurred in the most recent past and which had only limited and/or experimentally based evidences in older dedicated textbooks. Section III, with over 100 pages, represents the central core of the book dedicated to the early management of open spina bifida, the most interesting contributions being represented by the comprehensive chapter on radiological evaluation, the two chapters on primary surgical repair and related plastic surgery aspects and the one on the contribution endoscopy has given to the management of the associated hydrocephalus. The following four sections (Sections IV to VII) fully detail the postoperative multidisciplinary care of the child with open spina bifida including the management of spasticity, seizures, vertebral and extremities orthopaedic deformities, urological problems, rehabilitation, endocrinological and psychosocial aspects. Finally, Section VIII, with over 80 pages, is devoted to occult spinal dysraphism, including the combined management of anterior meningoceles, an impressively referenced (369 citations) paper on spinal lipomas as well as very well-imaged chapters on neurenteric cysts, diastematomyelia and dermal sinuses. In conclusion, the book which is supported by the all over high-quality reproduction of images represents a standard of care collection which every involved physician should have the will to consult in the management of this kind of disease. 